Research Article

\title{
Intelligent Assistant Decision-Making Method for Power Enterprise Customer Service Based on IoT Data Acquisition
}

\author{
Rui Xu (D), Dan Long, Jia Liu, Wanghong Yu, and Lei Xu \\ Power Customer Service Centre of Yunnan Power Grid Co. Ltd., Kunming, Yunnan, China \\ Correspondence should be addressed to Rui Xu; xurui@yccxip.com
}

Received 28 June 2021; Accepted 24 July 2021; Published 6 August 2021

Academic Editor: Fazlullah Khan

Copyright $\odot 2021$ Rui Xu et al. This is an open access article distributed under the Creative Commons Attribution License, which permits unrestricted use, distribution, and reproduction in any medium, provided the original work is properly cited.

\begin{abstract}
The prevailing era of the Internet of Things (IoT) has renewed all fields of life in general, but, especially with the advent of artificial intelligence (AI), has drawn the attention of researchers into a new paradigm of life standards. This revolution has been accepted around the world for making life comfortable with the use of intelligent devices. AI-enabled machines are more intelligent and capable of completing a specific task which saves a lot of time and resources. Currently, diverse methods are available in the existing literature to handle different issues of real life based on AI and IoT systems. The role of decision-making has its prominence in the AI-enabled and IoT systems. In this article, an AI- and IoT-based intelligent assistant decision-making method is presented for power enterprise customer service. An intelligent model of the customer service data network is designed, and the method of collecting data from IoT to assist decision-making is presented. Then, the semantic relationship of customer service data is defined, and the sharing scope of data transmission and resources are determined to realize intelligent assistant decisionmaking of customer service in power enterprises. Simulation results show that the proposed method improves the decision data transmission speed and shortens the transmission delay, and the network performance of data interaction is better than that of the existing methods.
\end{abstract}

\section{Introduction}

AI-enabled devices are more intelligent and capable of completing a specific task which saves a lot of time and resources. The use of IoT and network provides the principal way out due to less cost and adaptable features [1]. The main function of IoT is to provide links to the available resources with effectiveness and reliability. IoT is composed of three main components: digitization of resources, collection of data about the resources, and computational algorithms to control the system formed by the interconnected resources [2]. The IoT has several massive applications in real life which make life easier and comfortable. Various methods, techniques, tools, and approaches are available to handle different problems of real life based on AI and IoT systems. The role of decision-making has its significance in the AIenabled and IoT systems [3-5].

Several methods are available for AI-enabled decisionmaking in the system of IoT.
An intelligent decision-making system based on IoT was presented in [6]. The system architecture was based on two steps, and the criteria were devised for the selection of each part of the system. The performance of the system was validated with a case study of temperature monitoring. Chatfield and Reddick [7] designed a framework of smart government performance decision-making systems based on IoT-enabled techniques. The framework was functional for conducting the study analysis of IoT cybersecurity policy at the US federal government level. Gill et al. [8] analyzed the impacts of artificial intelligence, blockchain, and IoT on future cloud computing systems. The authors in [9] conducted a study to identify, perform, and evaluate approaches of AI for securing the environment of IoT. Parda [10] presented a study on the applications of IoT in decisionmaking about the assessment and management of pain in different organs of the human body. Hansen et al. presented a thorough investigation on $\mathrm{AI}$ and IoT for discovering the existing opportunities and shortcomings for enabling 
predictive analytics. An overview of the IoT and AI-enabled system along with four capabilities are provided, and then the review of the literature and its analysis was shown. $\mathrm{Du}$ et al. [11] presented an intelligent decision strategy for the iron ore sintering process based on abnormal condition prediction. Initially, the model of running mode prediction was established by using the fuzzy rules model, and the input was selected by one-way ANOVA. Based on this, the intelligent decision strategy of running parameters was proposed. The experiment was carried out with the actual operational data collected from the industrial field. The innovation of their model lies in the establishment of a prediction model based on fuzzy rules and the design of intelligent decision strategies based on priority to improve the abnormal operation mode. However, this approach has poor throughput performance. Niyama and colleagues [12] used a competitive ecosystem model, the competitive Lotka-Volterra (LV) model, to demonstrate the identification mechanism of the decision-making process. Based on the winner-take-all mechanism in the competitive LV model, the nonoptimal selection was eliminated and only the optimal selection was selected. In addition, the mean-field approximation mechanism was applied to the proposed decision method, and it was proved that the proposed method has good scalability as compared to data selection. Bandaragoda et al. [13] conceptualized, designed, and developed an AI-based commuter behavior profiling framework to predict different commuter behavioral profiles and fluctuating and routine patterns among commuters using traffic flow profiling and travel trajectory analysis. Their system was capable of real-time decision-making for road infrastructure and decision-making of government and business entities to optimize operations. An IoT-inspired framework has been designed for real-time analysis of athlete performance [14]. IoT data was employed to quantify athlete performance in the terms of probability parameters of probabilistic measure of performance and level of performance measure. The proposed model showed improved performance in terms of temporal delay, classification efficiency, and reliability.

Decision support technology [15] including computer, artificial intelligence, multimedia network, and other high technologies, artificial thinking, and decision calculation complement each other by assisting and supporting the organization of decision-making activities or businesses. With the rapid development of communication technology, decision-making activities have entered a new era. In the scientific and programmed stage, intelligent decision support technology emerges spontaneously. By the obvious advantages of qualitative analysis and uncertain reasoning [16], the technique makes full use of empirical knowledge, further penetrates the fusion of artificial thinking and decision computing, and is widely used in various fields. Therefore, based on the data acquisition method of the Internet of Things, this paper studies the intelligent decisionmaking method for customer service centre data sharing.

The intelligent service centre is mainly used for product consultation, feedback, presale, and customer protection and has been widely used in the field of finance and telecommunications [17]. In the case of a large number of consumers, some enterprises use the tools of intelligent service centres, but due to the limitation of technical level, for some intelligent services, the information is inaccurate and the service efficiency is low. To make intelligent decisions, it is necessary to provide accurate information resources according to the needs of customers [18]. Considering that the customer service centre is still a new field in the electric power enterprise, and the electric power enterprise is an important part of the national economy leapfrog. In this study, an AI- and IoT-based intelligent assistant decision-making system is proposed for power enterprise customer service centres. We designed an intelligent model of the customer service data network and devised the method of collecting data from IoT to assist the decision-making process. Furthermore, the semantic relationship of customer service data was identified, and the scope of data transmission was determined to realize intelligent assistant decision-making of customer service in power enterprises.

The paper is organized as follows. Section 2 provides a framework of the customer service data network. Section 3 describes the cyclic neural network and backpropagation method for developing an intelligent decision-making system of power customer service enterprise. In Section 4, different experimental results are presented. Finally, the paper is concluded in Section 5.

\section{Customer Service Data Network Model}

To bring the technical value of the customer service data model of electric power enterprises into full function, the network model of customer service data of electric power was designed based on the data collection terminals of the Internet of Things [19] to realize data integration and management and resource sharing of customer service data of electric power enterprises on the cloud. The customer service data network model is shown in Figure 1.

The model framework is composed of an extended application module, a model module, a data module, a data source, and related software. The main functions of each module are described as follows:

(i) Extended Application Module. This module presents an interface for electric power enterprise users to operate through web browsers. After the user completes the interface login process on the portal site, they can get the relevant data in real time. The role of the extended application module is to share customer service model data of power enterprises using the IoT techniques [20].

(ii) Model Module. The function of this module is to manage module browsing and database background services. The former presents a customer service data model by rendering technology, and the latter realizes dynamic management by managing data and cooperating with customer service participants. At the development stage, the model module should focus on three key points: reducing the file memory, 


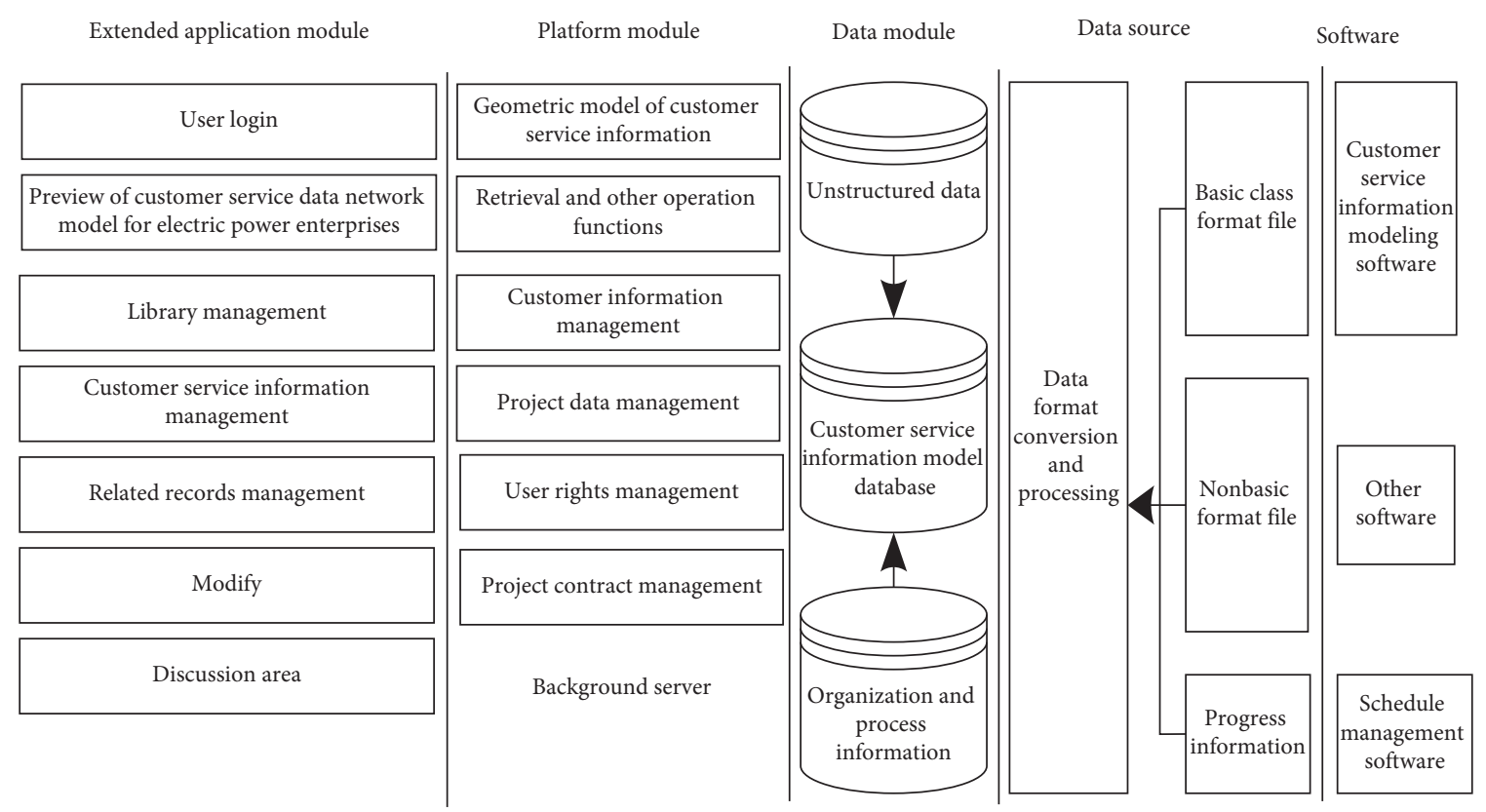

FIgURE 1: Schematic framework of customer service data network model of electric power enterprises.

ensuring the display quality and operation speed of the customer service data model of the power enterprise in the web page; improving the data retrieval speed; and providing different login accounts and management authority for the user role to ensure that different users on the customer service data network model of the power enterprise can perform data sharing safely.

(iii) Data Module. This module adopts different storage management methods for each data type. The data of the customer service model of structured power enterprises are managed using basic standards [21], the data of unstructured documents are stored by the document management system, and the data of organization and process are managed by the corresponding database. The function of the module is to store the basic data, customer service model data, and engineering data.

(iv) Data Source. This module is used to convert multiclass initial data into an industrial basic standard format, reduce the complexity of data queries, and provide data sharing and other functions.

\section{Proposed Model}

Due to the complexity of customer service structure and the uncertainty of customer demand in power enterprises, a cyclic neural network with strong fuzzy data processing capability was designed [22] to realize intelligent decision support for customer service in power enterprises.

3.1. Cyclic Neural Network Model. Using the nonlinear transformation unit of a recurrent neural network (RNN) neuron, the backpropagation training model was created.
Backpropagation is the core of training a neural network. It is the method of tuning the weights of a neural network based on the error rate received in the previous epoch. Proper fine-tuning of the weights allows you to decrease the error rates and make the model reliable by increasing its generalization [23]. The recurrent neural network also called a circular neural network can traverse the data elements inside a sequence and save the current state every time an element is traversed, as the input state of the next iteration. The basics structure of the neural network is shown in Figure 2.

The optimization and fine-tuning of the circular neural network depends upon the following factors:

(i) Number of Hidden Layers. The number of hidden layers is determined according to the complexity of the nonlinear problem to be simulated. Usually, if there is only one hidden layer, the number of nodes in the network is large enough to deal with relatively complicated problems.

(ii) Number of Nodes of the Hidden Layer. Deciding the number of nodes in the hidden layers is a very significant part of deciding your overall neural network architecture. Although hidden layers do not directly interact with the external environment, they have a great effect on the final output. Both the number of hidden layers and the number of neurons in each of these hidden layers must be carefully considered.

(iii) The Number of Nodes of the Input Layer. The number of nodes of the input layer shall be determined according to the number of main influencing factors of the problem to be solved [24].

(iv) The Number of Nodes of the Output Layer. These nodes greatly reduce the number of problems that 


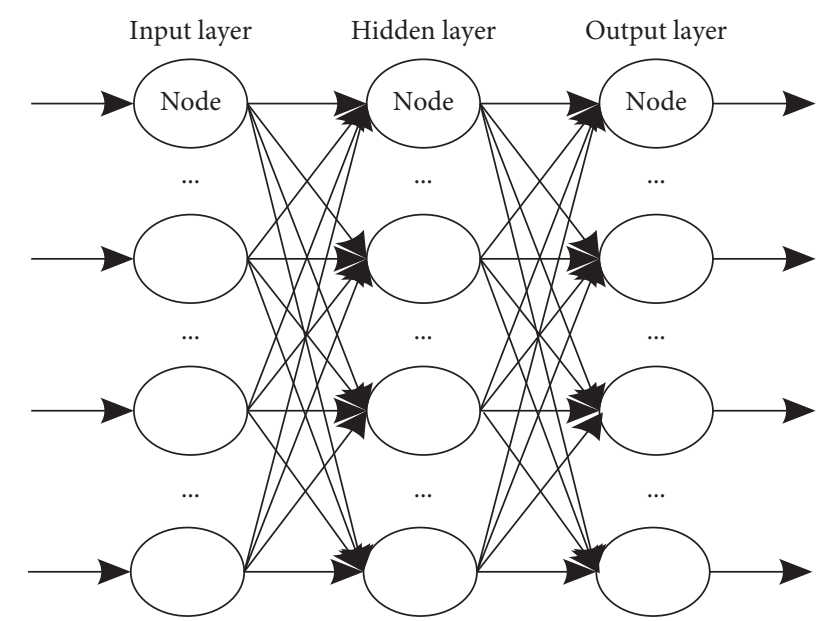

FIGURE 2: Basic diagram of the backward-propagation neural network.

need to be completed in one iteration and take the number of problems that need to be completed as the number of nodes of the output layer.

(v) Transformation Function. The sigmoid function with gradient search function is selected as the network transformation function, and its monotonously increasing nonlinear function curve was used to reflect the property of neuron saturation.

(vi) Sample Selection and Normalization. The samples are selected based on the level of accuracy. To ensure that the variables can be effectively input to the network, input variables are normalized in the range of 0 and 1 . Since the sigmoid function tends to infinity at intervals of $a$ and $b$, the convergence time is usually long. Therefore, the normalized range of the sample is set in the domain other than the two intervals.

3.2. Neural Network Model Learning. To design the proposed intelligent decision-making system, the neural network was trained using the backward-propagation training technique. The model was also optimized using different hyperparameters. Before the explanation of the backpropagation process, the forward propagation process of the neural network is described in the following section.

3.2.1. Forward Propagation Supervised Learning Algorithm. Taking a four-layer neural network as an example, the forward propagation steps in its learning algorithm are described as follows:

(i) Assuming the existence of $N$ nodes in the input layer, equalize the input and output of any node $i$; namely,

$$
x_{i}=O_{i},
$$

where $x_{i}$ represents the input of the input layer node $i$ and $O_{i}$ represents the output of that node. (ii) Assuming that the number of nodes in the first hidden layer is $N^{\prime}$, the connection weight between the node $j$ of the hidden layer and node $i$ of the input layer is $w_{i j}$, the threshold value of the node $j$ is $\theta_{j}$, and the sigmoid type function is $f(\cdot)$, then the expressions of input $x_{j}$ and output $O_{j}$ of node $j$ of the first hidden layer are as calculated using the following equation:

$$
\begin{gathered}
x_{j}=\sum_{i=1}^{N} w_{i j} O_{i}+\theta_{j}, \\
O_{j}=f\left(x_{j}\right)=\frac{1}{1+e^{-x_{j}}} .
\end{gathered}
$$

(iii) If the number of nodes of the second hidden layer is known to be $N^{\prime \prime}$, the following equations are used to solve the input $x_{k}$ and output $O_{k}$ of any node $k$ :

$$
\begin{gathered}
x_{k}=\sum_{j=1}^{N^{\prime}} w_{j k} O_{j}+\theta_{k}, \\
O_{k}=f\left(x_{k}\right)=\frac{1}{1+e^{-x_{k}}},
\end{gathered}
$$

where $w_{j k}$ represents the connection weights between nodes $j$ and $k$ of the two hidden layers and $\theta_{k}$ represents the threshold of the node $k$.

(iv) If the number of nodes in the output layer is $N^{\prime \prime \prime}$, the threshold value of any node $l$ in the output layer is $\theta_{l}$, and the connection weight between the node $l$ and the node $k$ in the hidden layer is $w_{k l}$, then the equation of input $x_{l}$ and output $O_{l}$ of the node $l$ is as follows:

$$
\begin{gathered}
x_{l}=\sum_{k=1}^{N^{\prime \prime}} w_{k l} O_{k}+\theta_{l}, \\
O_{l}=f\left(x_{l}\right)=\frac{1}{1+e^{-x_{l}}} .
\end{gathered}
$$

(v) If the sample-invariant function is a square function, and $p_{l}$ out of $p$ samples are trained, and $l$ node value of $p_{l}$ training sample is $t_{l}^{p_{l}}$, and the operation result of this node after $p_{l}$ training is $O_{l}^{p_{l}}$, then the decision deviation $E$ can be obtained by computing the following equation:

$$
E=\frac{1}{2} \sum_{p_{l}}^{p} \sum_{l=1}^{N^{\prime \prime \prime}}\left(t_{l}^{p_{l}}-O_{l}^{p_{l}}\right)^{2} .
$$

\subsubsection{Backpropagation Supervised Learning Algorithm.} We adjusted the network weights according to the negative gradient direction of deviation $E$ and created the following backpropagation learning algorithm flow:

(i) If the network is trained $M$ times, the weight gain coefficient is $\eta$, the input to the node $k$ after $p_{l}$ 
training is $x_{k}^{p_{l}}$, and the degree between the node $k$ and the node is $l \delta_{k l}^{p_{l}}=\left(t_{l}^{p_{l}}-O_{l}^{p_{l}}\right) O_{l}^{p_{l}}\left(1-O_{l}^{p_{l}}\right)$; the new connection weight $w_{k l}^{\prime}$ between the output layer and the second hidden layer and the new threshold $\theta_{l}^{\prime}$ of the node $l$ is obtained using the following equation:

$$
\begin{aligned}
w_{k l}^{\prime}(M+1) & =w_{k l}(M)+\eta \sum_{p_{l}=1}^{p} \delta_{k l}^{p_{l}} x_{k}^{p_{l}}, \\
\theta_{l}^{\prime}(M+1) & =\theta_{l}(M)-\eta \sum_{p_{l}=1}^{p} \delta_{k l}^{p_{l}}
\end{aligned}
$$

(ii) If the internode degree value is $\delta_{j k}^{p_{l}}=\sum_{l=1}^{N^{\prime \prime}} \delta_{k l}^{p_{l}} w_{k l}^{\prime} x_{k}^{p_{l}}\left(1-x_{k}^{p_{l}}\right)$, the new hidden layer node $k$ threshold $\theta_{k}^{\prime}$ and node connection weight $w_{j k}^{\prime}$ are as follows:

$$
\begin{gathered}
w_{j k}^{\prime}(M+1)=w_{j k}(M)+\eta \sum_{p_{l}=1}^{p} \delta_{j k}^{p_{l}} x_{j}^{p_{l}}, \\
\theta_{k}^{\prime}(M+1)=\theta_{k}(M)-\eta \sum_{p_{l}=1}^{p} \delta_{j k}^{p_{l}}
\end{gathered}
$$

(iii) After the degree value between the input layer node $i$ and the first hidden layer node $j$ is obtained by solving equations (8)-(10), they are adopted to solve the new connection weight $w_{i j}^{\prime}$ and the new threshold $\theta_{j}^{\prime}$ of the hidden layer node between the two nodes:

$$
\begin{aligned}
\delta_{i j}^{p_{l}} & =\sum_{k=1}^{N^{\prime \prime \prime}} \delta_{j k}^{p_{l}} w_{j k}^{\prime} x_{j}^{p_{l}}\left(1-x_{j}^{p_{l}}\right), \\
w_{i j}^{\prime}(M+1) & =w_{i j}(M)+\eta \sum_{p_{l}=1}^{p} \delta_{i j}^{p_{l}} x_{i}^{p_{l}}, \\
\theta_{j}^{\prime}(M+1) & =\theta_{j}(M)-\eta \sum_{p_{l}=1}^{p} \delta_{i j}^{p_{l}} .
\end{aligned}
$$

According to the characteristics of the network, the decision facts are divided into nonsequential values and sequential values based on the network values. Among the factors affecting the decision support, the nonsequential value includes the precision of the customer service model, knowledge integrity, performance, and innovative technology, and the sequential value includes the project price.

\subsection{Model Optimization}

3.3.1. Data Node Transfer Protocol. To ensure the accuracy of the proposed customer service intelligent decision-making model, the data node transmission protocol was designed. The first node specifies the starting point of the network connection and, based on the broadband characteristics, determines the profile of the leaf node or the service node to obtain the node address data in the network after the node is connected to the network. It also establishes and maintains the transmission with the nearest node based on the knowledge of other nodes, forwards the updated business resource intelligence data to the business node in real time, and then the service neighbour node sends the updated resource data. To adapt to the dynamic change of the intelligent service in the resource, when the leaf node connects to the service node that needs to connect to the resource, the leaf node exits the network. Service nodes receive messages, send output messages, disconnect from table nodes, delete resource pointers that need to update table data, and subtract the number of service table nodes [25]. In addition, if a leaf node does not send an update message to the service node within a specified time interval, it will detect a leaf node failure. In this case, the service node sends a message to the checklist node. If no response is received, the output of the leaf node is generated according to the above procedure. Based on the LEACH protocol, in the case of resource sharing requests, from the perspective of effectively organizing resources, it allows nodes to send connection requests to service nodes according to the LEACH protocol strategy and allows service nodes to share resources among data nodes, transmission requests, and neighbour service nodes and transmit resource sharing requests. When the leaf node finds that the connection to the service node does not work, the structure of the network is self-organized, so the leaf node supports a logical neighbourhood topology, and a request is sent to the shared service of another model so that the node can find the shared result in the adjacent region. Power enterprises' customer service data are processed as the core of transmission between nodes, through the exchange of data in the header, recording the relevant routing data [26]. The message type and description of the header are shown in Table 1, where the type is sorted in the positive order of the header. The receiver node is added to the routing list according to its situation to respond to the transmission request, and then the description of the node transmission protocol in the model is completed.

3.3.2. Effective Decision Data Interaction Based on Semantic Matching. To avoid data heterogeneity, the semantic matching algorithm was used to explain and share heterogeneous data and realize the interaction of effective decision data between nodes. The model adopts the data interaction mode of mixed ontology, constructs the metadata ontology of customer service data resources of electric power enterprises, and creates the standard vocabulary of data resources. It gives out the consistent data semantics representation of all nodes and finds out the concepts with the same or similar semantics in mapping semantics among metadata ontologies and resource metadata ontologies of all nodes [27]. Moreover, it also defines vocabulary through linguistic ontology, expresses concept names and attribute names, divides vocabulary relations, determines the corresponding relationship weights, and quantifies the relevance degree between node words. The details of association weights are given in Table 2 . 
TABLE 1: Message type and description of the data transfer message header.

\begin{tabular}{lcc}
\hline Sort by type & Message type & Definition \\
\hline 1 & ID & Message identifier to identify customer service data of power enterprises \\
2 & ID address & The IP address of the message sender \\
3 & Type & Identify the type of message \\
4 & Route & Routing data \\
5 & Hops & Message forwarding times \\
6 & Active & The node detects whether the neighbour node is connected \\
7 & Connect & Adjust the topology connection of the initialization node \\
8 & Attach & Response of connecting adjustment command \\
9 & Search hit & Data resource sharing request \\
10 & Name & Identifies the sender of the message \\
11 & Port & Communication port of message sender \\
12 & Community & Determine where to send the message \\
13 & TTL & The number of times a message can be forwarded \\
14 & Payload & Message additional data \\
15 & OK & Notification node online \\
16 & Search & Data resource sharing request-response \\
\hline
\end{tabular}

Based on Table 2, the reachable path correlation strength $A$ of terms in the semantic network is obtained as follows:

$$
A=a_{12} \times a_{23} \times \cdots \times a_{m(m-1)},
$$

where $a_{i j}$ is the associated weight and $m$ represents the length of the reachable path. Assume that there are several paths between words, the maximum correlation intensity in the path is chosen as the similarity $H$ and is computed as

$$
H=C_{i j}-C_{i j} \times W_{i j} \text {, }
$$

where $W_{i j}$ represents the metadata ontology similarity of words $i$ and $j$ and $C_{i j}$ represents similarity. The semantic relationship similarity $Q$ can be calculated as

$$
Q=1-\left|a_{J}-a_{n}\right|
$$

where $a_{J}$ and $a_{n}$ represent the weights of the semantic relations $J$ and $n$, respectively.

In this study, the semantic matching algorithm was employed to classify the weight of semantic association according to the similarity of lexical names, contexts, and semantic relations and to map the semantics satisfying the conceptual conditions according to different associations' concepts. Moreover, it was applied to determine the query scope of customer service data resources of power enterprises, locate shared data resources, and then transmit interactive decision data by nodes.

\section{Experimental Demonstration and Analysis}

The proposed method was compared with the two common methods of customer service data-aided decision-making mentioned in the Introduction section (method 1 and common method $2[10,11]$, resp.) to compare the throughput performance and transmission efficiency of data sharing.

4.1. Experimental Preparation. The customer service centre of an electric power enterprise was selected as the experimental object. The enterprise is a comprehensive enterprise
TABLE 2: Association weights for the node vocabulary.

\begin{tabular}{lcc}
\hline Node vocabulary & Relationship & Correlation weight \\
\hline \multirow{4}{*}{ Language features } & Synonym & 0.2 \\
& Superordinate words & 0.5 \\
& Hyponym & 1.0 \\
& Abbreviations & 0.3 \\
\hline \multirow{5}{*}{ Semantic features } & Be equal to & 0.8 \\
& Be similar to & 0.9 \\
& Part & 0.4 \\
& Contain & 0.2 \\
& Cover & 1.0 \\
\hline
\end{tabular}

of the electric power industry, including nuclear power plants, thermal power plants, coal-fired power plants, and other production projects. The actual data of the tools, consumables, and supply chain of the enterprise were simulated to obtain the metadata contributing resources. In the stand-alone environment, this study simulated the decision-making process of customer service data, including sharing resource design, resource uploading, resource sending, and resource consensus. The simulation parameters set are shown in Table 3.

We used MyEclipse10 as the developmental environment, XACML as the description language, and Java 1.8.0 as the running environment. A PC with $618 \mathrm{MB}$ RAM and a 4.0 $\mathrm{GHz} \mathrm{CPU}$ was used for simulating the proposed decision-making system. The service node, leaf node, and document data were extracted randomly, and each document was uniquely identified for data-aided decisionmaking.

\subsection{Experimental Results}

4.2.1. Throughput. To examine the throughput of the proposed intelligent decision-making system for power enterprises, we set the maximum sharing distance of customer service centres nodes of electric power enterprises to $250 \mathrm{~m}$. The number of customer service nodes of electric power 
TABLE 3: Simulation parameter setting.

\begin{tabular}{lc}
\hline Parameter & Value \\
\hline Simulation scene size & $1500 \mathrm{~m} \times 1500 \mathrm{~m}$ \\
Channel type & Telecommunications \\
Working frequency & $6.297 \mathrm{GHz}$ \\
Minimum competitive window & Twenty \\
Number of service nodes & Twenty \\
Document resources & 53820 \\
Data record start time to end time & 15 days \\
Network interface type & Wireless text \\
Channel bandwidth & $12 \mathrm{MHz}$ \\
Simulation time & $45 \mathrm{~min}$ \\
Maximum competitive window & One thousand one hundred and fifty \\
The number of times a message can be forwarded & Seven \\
Number of leaf nodes & Two thousand and five hundred \\
Record data volume & 250 groups/day \\
\hline
\end{tabular}

enterprises was adjusted so that the sparsity of customer service nodes might directly affect the stability of the network. During sharing data resources, we compared the data throughput of all three methods using the number of customer service nodes of different electric power enterprises. The experimental results are shown in Figure 3.

As can be seen from Figure 3, when the number of customer service centre nodes in the power enterprise is increased, the data resource sharing path and data interaction volume are also increased. If the route establishment is more perfect, the data throughput will increase, and the data throughput will reach its peak at the number of 40 nodes when the network resources are fully utilized. If the network resources are relatively blocked, the throughput decreases again. However, the throughput of the proposed method is still higher than that of the two common methods. The average throughput of the proposed method is $4.56 \mathrm{M} / \mathrm{s}$, which is higher than the average throughput of the common methods 1 and 2, i.e., $3.78 \mathrm{M} / \mathrm{s}$ and $3.45 \mathrm{M} / \mathrm{s}$, respectively.

When the number of customer service centre nodes of power enterprises was set to 40, and the sharing distance between the nodes was also changed, the sharing distance directly affected the number of message forwarding, the number of adjacent nodes, and the competition intensity of shared channel. The experimental results are given in Figure 4.

As evident from Figure 4, when the distance of customer service nodes of power enterprises is too small, the collision probability, message forwarding times, and message hops increase, and the network throughput is low. When the distance of customer service nodes of power enterprises is too large, the transmission nodes will decrease rapidly, the network resources will not be fully utilized, and the network throughput will decrease accordingly. However, the throughput of the proposed method is higher than that of the two common methods, the average throughput is $4.56 \mathrm{M} / \mathrm{s}$, and the average throughput of the common methods 1 and 2 is $3.52 \mathrm{M} / \mathrm{s}$ and $3.62 \mathrm{M} / \mathrm{s}$, respectively.

4.2.2. Transmission Delay. We compared the data transmission delay of all three methods using a different number of customer service nodes. The comparison results are illustrated in Figure 5.

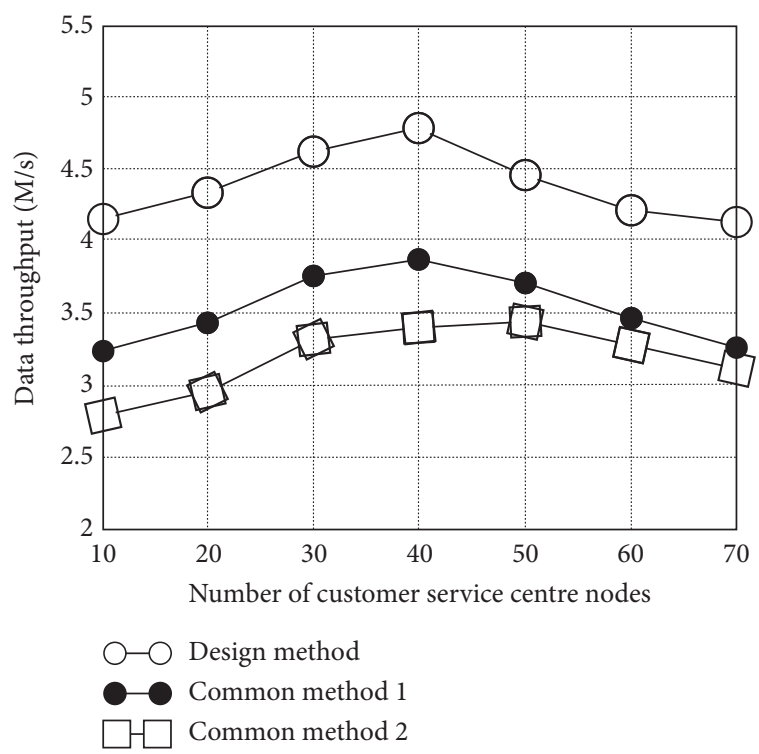

FIgURE 3: Throughput comparison results at different console node densities.

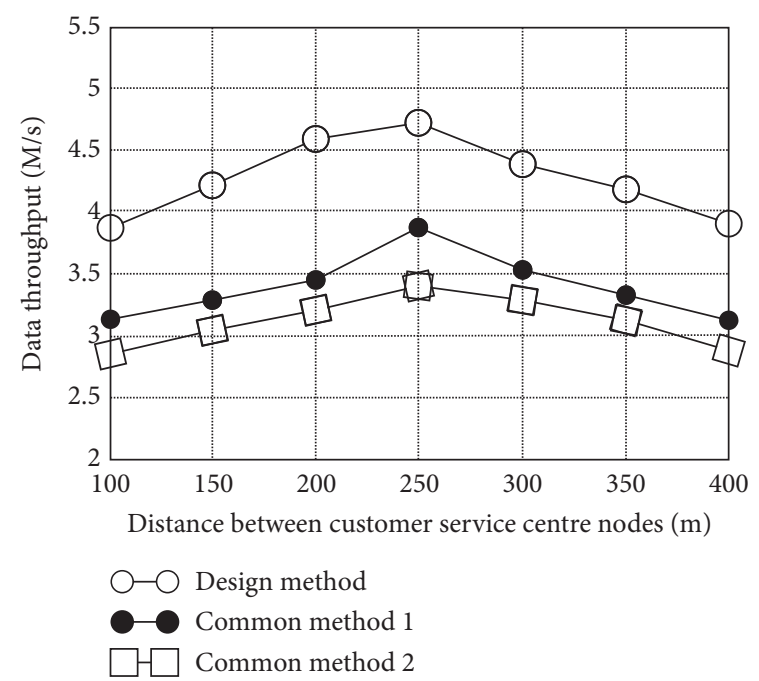

FIgURE 4: Throughput comparison results at different console node distances. 


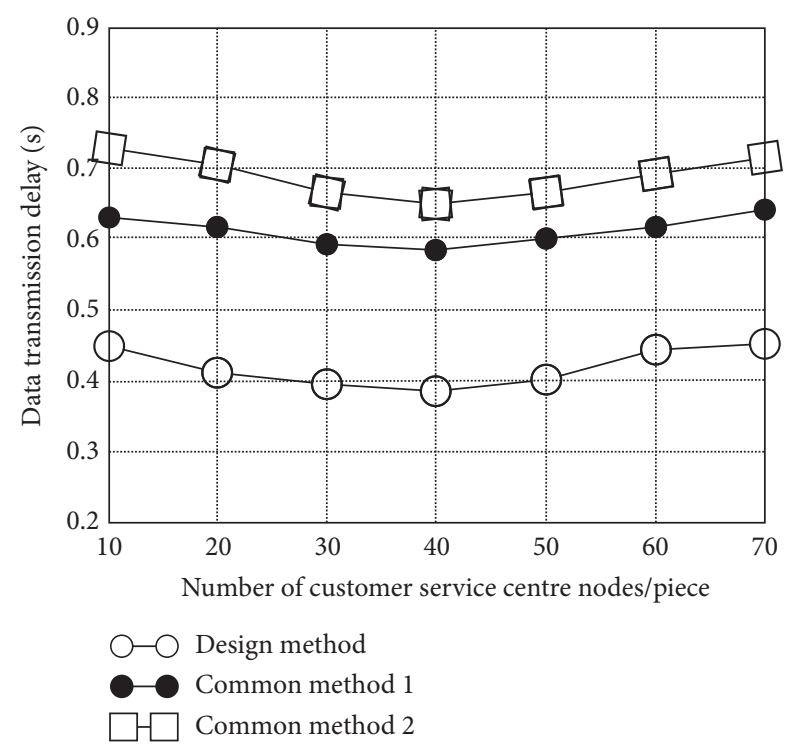

Figure 5: Transmission delay under different node densities.

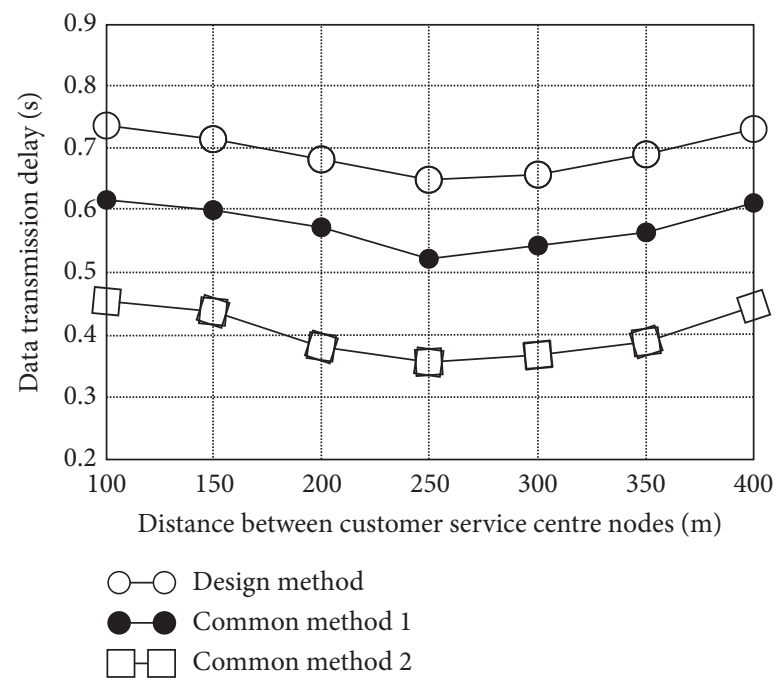

Figure 6: Transmission delay at different platform nodes.

With increasing the number of customer service centre nodes, the data transmission delay becomes irregular. However, the transmission delay of the proposed method in all cases is less than the other two methods. The proposed method reported the lowest average transmission delay of $0.41 \mathrm{~s}$ as compared to delay of $0.63 \mathrm{~s}$ and $0.69 \mathrm{~s}$ observed in the case of method 1 and method 2, respectively.

Figure 6 shows the effect of increasing distance between customer service centre nodes on transmission time.

It is evident that the average delay of data transmission is $0.37 \mathrm{~s}, 0.60 \mathrm{~s}$, and $0.73 \mathrm{~s}$ for the proposed method and methods 1 and 2, respectively. This shows that the transmission delay of the proposed method is lower than the other two methods even if the distance between the two nodes in customer service centres is increased. This proves the superiority of the proposed method.

4.2.3. Transmission Rate. Several experiments are carried out to count the data transmission rates using the three different methods. The results are given in Table 4.

It can be seen from Table 4 that when data resources are shared using all the three methods, the average data transmission rate is for the proposed method is $278.34 \mathrm{bit} / \mathrm{s}$, 
TABle 4: Data transmission rate comparison results (bit/s).

\begin{tabular}{lccc}
\hline Sharing times & & $\begin{array}{c}\text { Transmission rate } \\
\text { Common method 1 }\end{array}$ & 219.93 \\
\hline 10 & 279.39 & 236.17 & 227.69 \\
20 & 284.04 & 231.71 & 223.44 \\
30 & 272.10 & 240.49 & 211.20 \\
40 & 270.41 & 238.06 & 220.98 \\
50 & 281.92 & 233.18 & 217.72 \\
60 & 284.78 & 248.93 & 229.61 \\
70 & 278.24 & 235.26 & 218.54 \\
80 & 276.83 & 244.72 & 215.38 \\
90 & 272.29 & 249.14 & 214.22 \\
100 & 283.35 & 231.18 & \\
\hline
\end{tabular}

and the average transmission rates of common methods 1 and 2 are $238.88 \mathrm{bit} / \mathrm{s}$ and $219.87 \mathrm{bit} / \mathrm{s}$, respectively. As compared to the other two methods, the transmission rates of the proposed method are increased by $39.46 \mathrm{bit} / \mathrm{s}$ and $58.47 \mathrm{bit} / \mathrm{s}$, respectively.

\section{Conclusion}

The role of decision-making has its prominence in the AIenabled and IoT systems.

In this article, an intelligent assistant decision-making method is proposed for power enterprise customer service. We designed an intelligent decision-making model of the customer service centre and devised the method of collecting data from IoT to assist in decision-making. The semantic relationship among the customer service data is defined, and the sharing scope of data transmission and resources are determined to realize intelligent assistant decision-making of customer service in power enterprises. Simulation results show that the proposed method improves the decision data transmission speed and shortens the transmission delay, and the network performance of data interaction is better than the existing methods. In our future work, we will provide more flexible and efficient interoperability specifications for the interaction and integration of transmission languages between nodes and enhanced data sharing and will improve the security and reliability of the functional components of the proposed system.

\section{Data Availability}

The data used to support the findings of this study are included within the article.

\section{Conflicts of Interest}

The authors declare that they have no conflicts of interest regarding the publication of this paper.

\section{References}

[1] Y. Liu, B. Cao, K. Ma, and J. Fan, "Improving the classification of call center service dialogue with key utterences," Wireless Networks, vol. 27, no. 2-3, pp. 3395-3406, 2021.
[2] T. Itoa, N. Kakimurab, and Y. Kobayashic, "Complexity of the multi-service center problem," Theoretical Computer Science, vol. 842, pp. 18-27, 2020.

[3] M. Bhatia, S. Kaur, S. K. Sood, and V. Behal, "Internet of things-inspired healthcare system for urine-based diabetes prediction," Artificial Intelligence in Medicine, vol. 107, Article ID 101913, 2020.

[4] N. R. Odom, J. M. Lindmar, J. Hirt, and J. Brunty, "Forensic inspection of sensitive user data and artifacts from smartwatch wearable devices," Journal of Forensic Sciences, vol. 64, no. 6, pp. 1673-1686, 2019.

[5] C. I. Ossai and N. Wickramasinghe, "Intelligent therapeutic decision support for 30 days readmission of diabetic patients with different comorbidities," Journal of Biomedical Informatics, vol. 107, Article ID 103486, 2020.

[6] W. Li and S. Kara, "Methodology for monitoring manufacturing environment by using wireless sensor networks (WSN) and the internet of things (IoT)," Procedia CIRP, vol. 61, pp. 323-328, 2017.

[7] A. T. Chatfield and C. G. Reddick, "A framework for internet of things-enabled smart government: a case of IoT cybersecurity policies and use cases in U.S. federal government," Government Information Quarterly, vol. 36, no. 2, pp. 346357, 2019.

[8] S. S. Gill, S. Tuli, M. Xu et al., "Transformative effects of IoT, Blockchain and artificial intelligence on cloud computing: evolution, vision, trends and open challenges," Internet of Things, vol. 8, Article ID 100118, 2019.

[9] S. Aldhaheri, D. Alghazzawi, L. Cheng, A. Barnawi, and B. A. Alzahrani, "Artificial immune systems approaches to secure the internet of things: a systematic review of the literature and recommendations for future research," Journal of Network and Computer Applications, vol. 157, Article ID 102537, 2020.

[10] E. J. Argüello Prada, "Internet of things (IoT) in pain assessment and management: an overview," Informatics in Medicine Unlocked, vol. 18, Article ID 100298, 2020.

[11] S. Du, M. Wu, L. Chen, W. Cao, and W. Pedrycz, "An intelligent decision-making strategy based on the forecast of abnormal operating mode for iron ore sintering process," Journal of Process Control, vol. 96, pp. 57-66, 2020.

[12] T. Niiyama, G. Furuhata, A. Uchida, M. Naruse, and S. Sunada, "Lotka-volterra competition mechanism embedded in a decision-making method," Journal of the Physical Society of Japan, vol. 89, no. 1, Article ID 014801, 2019.

[13] T. Bandaragoda, A. Adikari, R. Nawaratne et al., "Artificial intelligence-based commuter behavior profiling framework using Internet of things for real-time decision-making," 
Neural Computing \& Applications, vol. 32, no. 20, pp. 16057-16071, 2020.

[14] M. Bhatia, "Intelligent system of game-theory-based decision making in smart sports industry," ACM Transactions on Intelligent Systems and Technology, vol. 12, no. 3, pp. 1-23, Article ID 29, 2021.

[15] M. Fernandes, S. M. Vieira, F. Leite, C. Palos, S. Finkelstein, and J. M. C. Sousa, "Clinical decision support systems for triage in the emergency department using intelligent systems: a review," Artificial Intelligence in Medicine, vol. 102, Article ID 101762, 2020.

[16] J. Y. Kwak, C. Cho, Y. Shin, and S. Yang, "IntelliTC: intelligent inter-DC traffic controller for the internet of everything service based on fog computing," IET Communications, vol. 14, no. 2, pp. 193-205, 2020.

[17] M. W. Bi, Y. F. Bian, and M. Zuo, "Simulation research on BLSTM-PA for semantic analysis in food safety," Computer Simulation, vol. 37, no. 3, pp. 343-348, 2020.

[18] R. A. Shanab, S. A. Mohamed, N. A. Mohamed, and M. A. Attia, "Comprehensive investigation of vibration of sigmoid and power-law FG nanobeams based on surface elasticity and modified couple stress theories," Acta Mechanica, vol. 231, no. 9, pp. 1977-2010, 2020.

[19] X. Yu, Y. Chu, F. Jiang, Y. Guo, and D. Gong, "SVMs classification based two-side cross domain collaborative filtering by inferring intrinsic user and item features," KnowledgeBased Systems, vol. 141, pp. 80-91, 2018.

[20] K. Moreno-Sader, S. I. Meramo-Hurtado, and A. D. Gonzalez-Delgado, "Computer-aided environmental and exergy analysis as decision-making tools for selecting biooil feedstocks," Renewable and Sustainable Energy Reviews, vol. 112, pp. 42-57, 2019.

[21] X. Yu, D. Zhan, L. Liu, H. Lv, L. Xu, and J. Du, "A privacypreserving cross-domain healthcare wearables recommendation algorithm based on domain-dependent and domainindependent feature fusion," IEEE Journal of Biomedical and Health Informatics, 2021.

[22] P. Yuan, Y. Wang, M. Su, Z. Yang, and Q. Zhang, "Markov decision process-based routing algorithm in hybrid satellites/ UAVs disruption-tolerant sensing networks," IET Communications, vol. 13, no. 10, pp. 1415-1424, 2019.

[23] I. S. Amiri, J. Prakash, M. Balasaraswathi, V. Sivasankaran, and O. Henry, "Large-scale internet of things-based data aggregation backpressure routing for disaster management," Wireless Networks, vol. 26, no. 4, pp. 1-22, 2019.

[24] M. Yu, T. Quan, Q. Peng, X. Yu, and L. Liu, "A model-based collaborate filtering algorithm based on stacked autoencoder," Neural Computing and Applications, 2021.

[25] G. Kaihong, "Expected value from a ranking of alternatives for personalized quantifier," IEEE Intelligent Systems, vol. 34, no. 6, pp. 24-33, 2021.

[26] Z. Eliane, J. Victorelli, and D. Cesar, "Understanding humandata interaction: literature review and design recommendations," International Journal of Human-Computer Studies, vol. 134, pp. 13-32, 2020.

[27] D. Sonntag and H.-J. Profitlich, "An architecture of opensource tools to combine textual information extraction, faceted search and information visualisation," Artificial Intelligence in Medicine, vol. 93, pp. 13-28, 2019. 